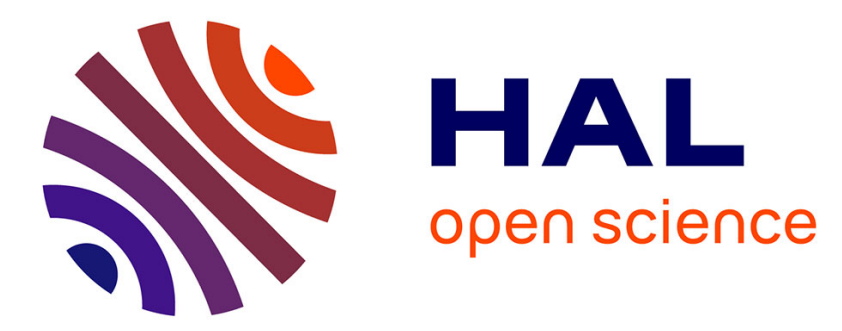

\title{
Trajectory planning/re-planning for satellite systems in rendezvous mission in the presence of actuator faults based on attainable efforts analysis
}

\author{
Abbas Chamseddine, Cédric Join, Didier Theilliol
}

\section{- To cite this version:}

Abbas Chamseddine, Cédric Join, Didier Theilliol. Trajectory planning/re-planning for satellite systems in rendezvous mission in the presence of actuator faults based on attainable efforts analysis. International Journal of Systems Science, 2015, 46 (4), pp.690-701. 10.1080/00207721.2013.797034 . hal-00831637

\section{HAL Id: hal-00831637 https://hal.inria.fr/hal-00831637}

Submitted on 7 Jun 2013

HAL is a multi-disciplinary open access archive for the deposit and dissemination of scientific research documents, whether they are published or not. The documents may come from teaching and research institutions in France or abroad, or from public or private research centers.
L'archive ouverte pluridisciplinaire HAL, est destinée au dépôt et à la diffusion de documents scientifiques de niveau recherche, publiés ou non, émanant des établissements d'enseignement et de recherche français ou étrangers, des laboratoires publics ou privés. 


\title{
Trajectory Planning/Re-planning for Satellite Systems in \\ Rendezvous Mission in the Presence of Actuator Faults Based on Attainable Efforts Analysis
}

\author{
Abbas Chamseddine, ${ }^{a}$ Cédric Join $^{b}$ and Didier Theilliol ${ }^{a}$ \\ ${ }^{a}$ Centre de Recherche en Automatique de Nancy (CRAN) - \\ CNRS UMR 7039 - University of Lorraine, \\ BP 70239 - 54506 Vandoeuvre Cedex - France \\ ${ }^{b}$ Centre de Recherche en Automatique de Nancy (CRAN) - \\ CNRS UMR 7039 \& ALIEN-INRIA - University of \\ Lorraine, BP 70239 - 54506 Vandoeuvre Cedex - France
}

\begin{abstract}
The objective of Fault-tolerant Control (FTC) is to minimize the effect of faults on systems performance (stability, trajectory tracking, etc.). However, the majority of the existing FTC methods continue to force the system to follow the pre-fault trajectories without considering the reduction in available control resources caused by actuator faults. Forcing the system to follow the same trajectories as before fault occurrence may result in actuator saturation and system's instability. Thus, pre-fault objectives should be redefined in function of the remaining resources to avoid potential saturation. The main contribution of this paper is a flatnessbased trajectory planning/re-planning method that can be combined with any active FTC approach. The work considers the case of over-actuated systems where a new idea is proposed to evaluate the severity of faults occurred. In addition, the trajectory planning/re-planning approach is formulated as an optimization problem based on the analysis of attainable efforts domain in fault-free and fault cases. The proposed approach is applied to two satellite systems in rendezvous mission.
\end{abstract}

\section{Keywords:}

Satellite systems, Trajectory planning/re-planning, Actuator faults, Flatness, Attainable efforts. 


\section{Introduction and problem statement}

Fault-tolerant Control (FTC) systems continue to gain more and more attention due to their importance in decreasing economic losses in industrial processes and preserving systems and operators safety. FTC systems can be classified into passive (PFTC) and active (AFTC) systems. In PFTC systems, controllers are fixed and are designed to be robust against a class of predefined faults (Hsieh, 2002). This approach needs neither fault detection and identification schemes nor controller reconfiguration, but it has limited fault-tolerant capabilities. On the other hand, AFTC systems (also referred to as self-repairing, reconfigurable or self-designing) react to the system component failures actively so that the stability and acceptable performance of the entire system can be maintained (Blanke et al., 2006; Noura et al., 2009; Dong et al., 2010). Various theoretical approaches have been proposed in the literature to accommodate faults based on linear quadratic, adaptive control, eigenstructure assignment or linear matrix inequality. In addition to the theoretical development, FTC has been applied to many fields such as aircraft engineering, underwater marine vehicles, chemical and petrochemical plants. For an overall picture of the FTC approaches and the application fields, the readers can refer to the bibliographical review of Zhang and Jiang (Zhang and Jiang, 2008) and the references therein.

Whatever the selected FTC strategy, a fault cannot be accommodated without sufficient resources in the system. However, almost all FTC strategies do not establish a relation between the reference trajectory to follow and the remaining resources after faults occurrence. In other words, the feasibility of the mission after faults is not investigated and no solutions are given when the predefined mission cannot be accomplished. Mission unaccomplishment occurs for example when actuators hit their limits and cannot deliver the actuation inputs desired by the controller. This has more tendency to take place when actuator faults occur and the system resources decrease. Control systems are often designed for linearized systems and do not directly consider amplitude limitations on the control inputs. Then, input bounds may not only lead to the unaccomplishment of the mission but also can be source of parasitic equilibrium points and limit cycles, or can even lead the closed-loop system to an unstable behavior (Henrion et al., 2001). Several works considered this problem either by avoiding saturation (Castelan et al., 1996), (Henrion et al., 2001) or allowing saturation while designing stabilizing controllers with saturating controls (Tarbouriech et al., 2002).

Reference management or reference governor is proposed in the literature for systems with input and/or state related constraints. In (Bemporad et al., 1997), a command governor based on conceptual tools of predictive control is designed for solving set-point tracking problems wherein pointwise-in-time input and/or state inequality constraints are present. A reference governor is designed in (Gilbert and Kolmanovsky, 2002) for general discrete-time and continuous-time nonlinear systems with uncertainties. It relies on safety properties provided by sub-level sets of equilibria-parameterized functions. A spline parametrisation is considered in (Suryawan et al., 2012) for trajectory planning of flat systems with constraints. With such parametrisation, the problem of constrained trajectory planning can be cast into a simple quadratic programming problem. In the context of FTC, a reference input management is introduced in (Zhang and Jiang, 2003) to determine appropriate reference inputs in the presence of 
actuator faults to avoid potential saturation. The idea is to determine the relationship between the closed-loop control signals and the associated reference inputs at steady state and to translate the limits of actuator saturation to the desired requirements on the reference inputs. In (Theilliol et al., 2009), an online adjustment strategy of reference input trajectories is developed using model predictive control techniques. Another reference inputs generation method is proposed in (Dardinier-Maron et al., 1999). The reference inputs generation which leads the damaged system to its optimal operating point corresponds to a nonlinear quadratic programming optimization problem. The objective is to minimize the distance between the desirable output vector before and after failure occurrence while distributing the most equitably the energy among the healthy actuators.

\section{Objectives and followed methodology}

When referring to the research works cited above, it can be noted that the input constraints are considered in the generation of the reference. However, they do not quantify explicitly the available resources in the system and they do not give a priori information about the feasibility of the desired reference trajectory. This paper carried out in the framework of SIRASAS project (SIRASAS, 2011), proposes a flatness-based method for trajectory planning/re-planning that can be combined with any AFTC approach. Three main advantages of flatness are considered to tackle such problem:

1. It allows computing a priori the nominal control inputs to be applied in function of the desired reference trajectory (Mai et al., 2007). This permits tuning the reference trajectory so that actuator constraints are not violated.

2. This a priori knowledge reduces calculation load and requirement since flatness-based trajectory planning can be performed only once before the beginning of the mission.

3. For the real-time trajectory generation, the flatness formulation considerably reduces the number of variables and constraints and this in turn can potentially lead to considerable reduction in computational time (Ross and Fahroo, 2006).

Flatness-based FTC has been introduced in (Mai et al., 2007) for a particular system and without any generalization where trajectory re-planning is employed to avoid systems from hitting their input saturation limits. This work proposes new ideas on flatness-based trajectory planning/re-planning when applied to over-actuated systems. The flatness-based trajectory planning/re-planning is posed as an optimization problem to minimize the total time of the mission while avoiding hitting actuator constraints. These ideas are presented by employing the example of satellite systems in rendezvous mission. It is worthy to note the following:

- The satellites' dynamics are nonlinear. However, during rendezvous phase, a linear model can be employed to represent the position dynamics of one satellite with respect to the other. This latter model is used for 
trajectory planning/re-planning design but simulations are carried out with the nonlinear models for more realistic results.

- The trajectory planning/re-planning is posed as an optimization problem where for simplification purposes the domain of attainable efforts is replaced by a sphere. As will be shown in the sequel, this sphere is determined based on a simplified domain which has fewer facets compared to the original domain.

- To account for model uncertainties, a parameter $\rho$ is introduced into the optimization problem to create a safety margin and to ensure that the desired efforts remain inside their attainable domain despite uncertainties.

- The reference trajectories are chosen as polynomial functions of degree five. It is shown later that when relative distances between satellites are relatively small, reference trajectories can be chosen as polynomial functions of degree three for a more suitable trajectory planning for on-board implementation with limited calculation capabilities. However, simulation results are only given for the former case.

While the objective is to minimize the total time of the mission, it should be pointed out that the obtained trajectories are suboptimal. This is because the reference trajectories are restricted to polynomial functions of time $t$ and second, the domain of attainable control efforts is replaced by a spherical subset.

\section{Satellite systems in rendezvous mission}

In this paper, two satellite systems in rendezvous mission are considered. The first one called target moves freely in an orbit while the second one called chaser is intended to join the target. The chaser and the target have the same position dynamics structure with the difference that the two spacecrafts do not have the same geometry (mass, drag coefficient, etc.) and that the chaser is equipped with thrusters which is not the case of the target. The position dynamics are:

$$
\begin{aligned}
& \ddot{x}_{\vartheta}=\frac{-\mu}{R_{\vartheta}^{3}}\left[1-\frac{1}{2} J_{2} R_{e q}^{2}\left(\frac{3}{R_{\vartheta}^{2}}-\frac{15 z_{\vartheta}^{2}}{R_{\vartheta}^{4}}\right)\right] x_{\vartheta}-\frac{\rho_{0}}{2 M_{\vartheta}} S_{\vartheta} C_{D_{\vartheta}} \bar{R}_{\vartheta} e^{\frac{R_{e q}-R_{\vartheta}}{H_{0}}} \dot{x}_{\vartheta}+\frac{1}{M_{\vartheta}} v_{x_{\vartheta}} \\
& \ddot{y}_{\vartheta}=\frac{-\mu}{R_{\vartheta}^{3}}\left[1-\frac{1}{2} J_{2} R_{e q}^{2}\left(\frac{3}{R_{\vartheta}^{2}}-\frac{15 z_{\vartheta}^{2}}{R_{\vartheta}^{4}}\right)\right] y_{\vartheta}-\frac{\rho_{0}}{2 M_{\vartheta}} S_{\vartheta} C_{D_{\vartheta}} \bar{R}_{\vartheta} e^{\frac{R_{e q}-R_{\vartheta}}{H_{0}}} \dot{y}_{\vartheta}+\frac{1}{M_{\vartheta}} v_{\vartheta_{\vartheta}} \\
& \ddot{z}_{\vartheta}=\frac{-\mu}{R_{\vartheta}^{3}}\left[1-\frac{1}{2} J_{2} R_{e q}^{2}\left(\frac{3}{R_{\vartheta}^{2}}-\frac{15 z_{\vartheta}^{2}}{R_{\vartheta}^{4}}\right)\right] z_{\vartheta}-\frac{\rho_{0}}{2 M_{\vartheta}} S_{\vartheta} C_{D_{\vartheta}} \bar{R}_{\vartheta} e^{\frac{R_{e q}-R_{\vartheta}}{H_{0}}} \dot{z}_{\vartheta}+\frac{1}{M_{\vartheta}} v_{z_{\vartheta}}
\end{aligned}
$$

with $R_{\vartheta}=\left(x_{\vartheta}^{2}+y_{\vartheta}^{2}+z_{\vartheta}^{2}\right)^{1 / 2}$ and $\bar{R}_{\vartheta}=\left(\dot{x}_{\vartheta}^{2}+\dot{y}_{\vartheta}^{2}+\dot{z}_{\vartheta}^{2}\right)^{1 / 2} x_{\vartheta}, y_{\vartheta}$ and $z_{\vartheta}$ represent the inertial coordinates of the satellite with respect to Mars. $\dot{x}_{\vartheta}, \dot{y}_{\vartheta}$ and $\dot{z}_{\vartheta}$ are the corresponding velocities. The subscript $\vartheta=\{c, t\}$ where $c$ and $t$ respectively stand for the chaser and the target. $v_{x_{c}}, v_{y_{c}}$ and $v_{z_{c}}$ are the efforts applied to the chaser. The target is uncontrolled and thus $v_{x_{t}}=v_{y_{t}}=v_{z_{t}}=0 . M_{\vartheta}$ is the satellite mass, $\mu$ and $R_{e q}$ are the gravitational constant and the equatorial radius of Mars, $J_{2}$ is the second zonal harmonic, $\rho_{0}$ is the atmospheric density 
at a reference altitude, $S_{\vartheta}$ is the shock surface of the spacecraft, $C_{D_{\vartheta}}$ is the drag coefficient and $H_{0}$ is a scale factor.

The position dynamics of the chaser and the target in (1) are complex and nonlinear. However, during the rendezvous mission the Clohessy-Wiltshire linear model can be employed to describe the relative position of the chaser with respect to the target. This linear model is given by:

$$
\begin{aligned}
& \ddot{x}_{1}=2 w \dot{x}_{2}+3 w^{2} x_{1}+v^{1} \\
& \ddot{x}_{2}=-2 w \dot{x}_{1}+v^{2} \\
& \ddot{x}_{3}=-w^{2} x_{3}+v^{3}
\end{aligned},
$$

where $x_{i}(i=1,2,3)$ represent the relative coordinates of the chaser with respect to the target. $w$ is the average motion. $v^{1}, v^{2}$ and $v^{3}$ represent the efforts respectively applied in the $x_{1}, x_{2}$ and $x_{3}$ directions. The relation between the applied efforts and the control inputs is given by $v(t)=B u(t)$ where $v=$ $\left[\begin{array}{lll}v^{1} & v^{2} & v^{3}\end{array}\right]^{T} \in \Re^{3}, u=\left[\begin{array}{lll}u^{1} & u^{2} \ldots u^{12}\end{array}\right]^{T} \in \Re^{12}$ and $B \in \Re^{3 \times 12}$. The control inputs are the forces generated by the chaser thrusters. They are bounded with $0 \leq u^{i} \leq 20 N$ and $i=1, \ldots, 12$. The satellite system is over-actuated and thus control allocation must be used to distribute the control inputs $u$ over the actuators given the desired effort $v^{*}(t)$. Among various control allocation methods (Petersen and Bodson, 2006), the direct allocation formulated as linear programming problem is used here (Bodson, 2002).

Remark 3.1 In the subsequent sections, the Clohessy-Wiltshire model (2) will be employed in the controller design as well as in the trajectory planning and replanning approach development. However, for more realistic simulation results, the developed approaches are applied to the nonlinear dynamics (1).

\section{$4 \quad$ Flatness and flatness-based control}

Flatness can be defined as follows (Gensior et al., 2006; Fliess et al., 1995). Let the system model be given by differential equations of the form:

$$
S_{i}\left(\mathcal{Z}, \dot{\mathcal{Z}}, \ddot{\mathcal{Z}}, \ldots, \mathcal{Z}^{\left(\sigma_{i}\right)}\right)=0, \quad i=1, \ldots, p
$$

where $\mathcal{Z}=\left(z_{1}, \ldots, z_{n}\right)$ are the system variables including also the input variables. Such a system is called (differentially) flat, if there exists an $m$-tuple $\mathcal{F}$ of functions:

$$
F^{i}=\psi_{i}\left(\mathcal{Z}, \dot{\mathcal{Z}}, \ddot{\mathcal{Z}}, \ldots, \mathcal{Z}^{\left(\eta_{i}\right)}\right), \quad i=1, \ldots, m
$$

fulfilling the following conditions:

1. The components of $\mathcal{F}$ are differentially independent, i.e., there does not exist a nontrivial differential equation of the form:

$$
\Xi\left(\mathcal{F}, \dot{\mathcal{F}}, \ddot{\mathcal{F}}, \ldots, \mathcal{F}^{(\alpha)}\right)=0
$$

which means such a relation in $\mathcal{F}$ only cannot be derived from $(3)$. 
2. The system variables in $\mathcal{Z}$ can be expressed by functions of $\mathcal{F}$ and its time derivatives:

$$
z_{i}=\phi_{i}\left(\mathcal{F}, \dot{\mathcal{F}}, \ddot{\mathcal{F}}, \ldots, \mathcal{F}^{\left(\gamma_{i}\right)}\right), \quad i=1, \ldots, n
$$

This implies that the trajectories of $\mathcal{F}$ provide a parameterization for the trajectories of the system variables (including the input variables).

The parameters $\sigma_{i}, \eta_{i}, \alpha, \gamma_{i}, p, m$ and $n$ in the equations above are positive integers. In the rest of the paper, the components of the $m$-tuple $\mathcal{F}$ are denoted as $F^{i}(i=1, \ldots, m)$ and are referred to as flat outputs. It can be shown that the position dynamics system (2) is differentially flat with the three flat outputs: $F^{1}=x_{1}, F^{2}=x_{2}$ and $F^{3}=x_{3}$. The differential parameterization of the efforts $v^{1}, v^{2}$ and $v^{3}$ in terms of the flat outputs is:

$$
\begin{aligned}
& v^{1}=\ddot{F}^{1}-2 w \dot{F}^{2}-3 w^{2} F^{1} \\
& v^{2}=\ddot{F}^{2}+2 w \dot{F}^{1} \\
& v^{3}=\ddot{F}^{3}+w^{2} F^{3}
\end{aligned} .
$$

Let us define $F^{i *}$ as the desired reference trajectory for the $i^{\text {th }}$ flat output $F^{i}$. To design an appropriate reference trajectory $F^{i *}$, a Bézier polynomial function of degree equals to five is considered. The reference trajectory for the $i^{\text {th }}$ flat output $F^{i}$ is given as follows:

$$
F^{i *}=a_{5}^{i} t^{5}+a_{4}^{i} t^{4}+a_{3}^{i} t^{3}+a_{2}^{i} t^{2}+a_{1}^{i} t+a_{0}^{i},
$$

The parameters $a_{j}^{i}(i=1,2,3$ and $j=0, \ldots, 5)$ are in function of the starting time $t_{0}$, the arriving time $t_{f}$, the initial conditions $F^{i *}\left(t_{0}\right), \dot{F}^{i *}\left(t_{0}\right), \ddot{F}^{i *}\left(t_{0}\right)$ and the final conditions $F^{i *}\left(t_{f}\right), \dot{F}^{i *}\left(t_{f}\right)$ and $\ddot{F}^{i *}\left(t_{f}\right)$. An important consequence of the above parameterization (7) is that once $F^{i *}$ are chosen, it is possible to determine the desired efforts $v^{i *}$ to be applied along the reference trajectory:

$$
\begin{aligned}
& v^{1 *}=\ddot{F}^{1 *}-2 w \dot{F}^{2 *}-3 w^{2} F^{1 *} \\
& v^{2 *}=\ddot{F}^{2 *}+2 w \dot{F}^{1 *} \\
& v^{3 *}=\ddot{F}^{3 *}+w^{2} F^{3 *}
\end{aligned}
$$

It can be seen that in steady state (i.e. $F^{i *}=\bar{F}^{i}=$ constant):

$$
\begin{aligned}
& v_{s s}^{1 *}=-3 w^{2} \bar{F}^{1} \\
& v_{s s}^{2 *}=0 \\
& v_{s s}^{3 *}=w^{2} \bar{F}^{3}
\end{aligned}
$$

From the differential parameterization (7), a flatness-based tracking control law can be synthesized when replacing $\ddot{F}^{i}$ by the auxiliary control input $\bar{v}^{i}(i=$ $1,2,3)$. Specifying $\bar{v}^{i}(i=1,2,3)$ as:

$$
\begin{aligned}
& \bar{v}^{1}=\ddot{F}^{1 *}+K_{11}\left(\dot{F}^{1 *}-\dot{F}^{1}\right)+K_{12}\left(F^{1 *}-F^{1}\right) \\
& \bar{v}^{2}=\ddot{F}^{2 *}+K_{21}\left(\dot{F}^{2 *}-\dot{F}^{2}\right)+K_{22}\left(F^{2 *}-F^{2}\right) \\
& \bar{v}^{3}=\ddot{F}^{3 *}+K_{31}\left(\dot{F}^{3 *}-\dot{F}^{3}\right)+K_{32}\left(F^{3 *}-F^{3}\right)
\end{aligned},
$$


ensures the tracking errors $e^{i}=F^{i *}-F^{i}$ to asymptotically converge to zero for an appropriate choice of control gains $K_{i j}(i=1,2,3$ and $j=1,2)$. Finally, the control inputs are:

$$
\begin{aligned}
& v^{1}=\ddot{F}^{1 *}-2 w \dot{F}^{2}-3 w^{2} F^{1}+K_{11}\left(\dot{F}^{1 *}-\dot{F}^{1}\right)+K_{12}\left(F^{1 *}-F^{1}\right) \\
& v^{2}=\ddot{F}^{2 *}+2 w \dot{F}^{1}+K_{21}\left(\dot{F}^{2 *}-\dot{F}^{2}\right)+K_{22}\left(F^{2 *}-F^{2}\right) \\
& v^{3}=\ddot{F}^{3 *}+w^{2} F^{3}+K_{31}\left(\dot{F}^{3 *}-\dot{F}^{3}\right)+K_{32}\left(F^{3 *}-F^{3}\right)
\end{aligned}
$$

It can be noted that $v^{i}$ is function of the derivatives $\dot{F}^{i}(i=1,2,3)$ of the flat outputs. These can be derived using, for example, an algebraic derivative estimation (Mai et al., 2007) if necessary. On the other hand, the derivatives of $F^{i *}$ can be easily derived from the Bézier polynomial function.

\section{Attainable control inputs and efforts analysis}

Consider the relation $v(t)=B u(t)$ defined in Section 3 where $v(t) \in \Re^{3}$ and $u(t) \in \Re^{12}$. Since this relation is linear, matrix $B$ represents then a linear transformation from a space of dimension 12 into a space of dimension 3 . The domain of attainable control inputs and the domain of attainable efforts can be defined as follows. The domain of attainable control inputs is constituted of all the values that $u^{i}$ can take with $i=1, \cdots, 12$. This domain of dimension 12 is a hyper-cube denoted by $\Omega$ :

$$
\Omega=\left\{u \in \Re^{12}: 0 \leq u^{i} \leq 20\right\} \subset \Re^{12} .
$$

The domain of attainable efforts is constituted of all the values that $v^{i}$ can take with $i=1,2,3$. This domain denoted by $\Phi$ is the projection of $\Omega$ via matrix $B:$

$$
\Phi=\left\{v \in \Re^{3}: v=B u \quad \forall u \in \Omega\right\} \subset \Re^{3} .
$$

Figure 1(a) illustrates the domain $\Phi$. It also shows the largest sphere of centre $V_{s s}\left(v_{s s}^{1 *}, v_{s s}^{2 *}, v_{s s}^{3 *}\right)$ included in $\Phi$. The radius of the sphere is the minimal distance from $V_{s s}$ to the boundary of $\Phi$. Note that a desired effort $v^{*}(t)$ can be generated by the control inputs $u$ if $v^{*}(t) \in \Phi$ (Bordignon, 1996). If $v^{*}(t) \notin \Phi$ then $v^{*}(t)$ cannot be generated resulting in actuators saturation and in some cases to instability. Thus, a reference trajectory $F^{*}$ is said to be feasible if the system can be driven along this trajectory, i.e. $v^{*}(t) \in \Phi \forall t \in\left[t_{0}, t_{f}\right]$, where $t_{0}$ and $t_{f}$ are the initial and final time of the mission, respectively. The point $V_{s s}$ is then of great importance, because it is the minimal effort needed to keep the system in steady state and should always be inside $\Phi$.

\section{$6 \quad$ Flatness-based trajectory planning}

Flatness has been employed in trajectory planning for the quadrotor unmanned aerial vehicle: a method is presented in (Bouktir et al., 2008) to generate timeoptimal trajectories for the quadrotor system. In (Cowling et al., 2006), Cowling et al. present a quasi-optimal trajectory planner with a simple LQR path following controller. Using the differential flatness, the trajectory planning is posed as a constrained optimization problem in the output space. This section presents 
the approach of flatness-based trajectory planning for the satellite systems in rendezvous mission in both fault-free and fault cases. The trajectory planning is made possible thanks to the parameterization of the control inputs as functions of the flat outputs (Rudolph and Winkler, 2003; Gensior et al., 2006; Mai et al., 2007).

\subsection{Fault-free trajectory planning}

Two cases will be considered for the fault-free trajectory planning. The first is the general case where the reference trajectories are chosen as polynomial functions of degree five. The second is the special case where the relative distances between chaser and target are relatively small and the reference trajectories are chosen as polynomial functions of degree three. This latter case is more suitable for on-board implementation. However, only simulation results for the former case will be given.

\subsubsection{General case}

The system can be driven from an initial state $F_{0}$ to a final state $F_{f}$ if $v^{*}(t) \in \Phi$ $\forall t \in\left[t_{0}, t_{f}\right]$. Thus, the objective of trajectory planning is to define the trajectory to track such that the desired effort $v^{*}(t) \in \Phi$. This issue can be handled by using flatness. Generally, the final state $F_{f}$ should be attained as fast as possible and thus, the above trajectory planning problem can be formulated as follows:

$$
\left\{\begin{array}{l}
\text { Minimize } \quad t_{f}-t_{0} \\
\text { Subject to } v^{*}(t) \in \Phi \quad \forall t \in\left[t_{0}, t_{f}\right]
\end{array} .\right.
$$

As can be seen in Figure 1(a), $\Phi$ has an irregular shape. To handle the constraint in (15) with less computational effort, the domain $\Phi$ is replaced by the largest sphere containing the point $V_{s s}$ and included in $\Phi$. The sphere is chosen because of its simple geometric shape. The sphere radius is denoted by $R$ and its centre by $C$. Thus, problem (15) is reformulated as follows:

$$
\left\{\begin{array}{l}
\text { Minimize } t_{f}-t_{0} \\
\text { Subject to } \sum_{i=1}^{3}\left(v^{i *}(t)-C^{i}\right)^{2}<R^{2} \forall t
\end{array},\right.
$$

where $v^{i *}(t)$ (respec. $C^{i}$ ) is the $i^{t h}$ component of $v^{*}(t)$ (respec. $C$ ). This new constraint imposes that the distance between $C$ and $v^{*}$ is smaller than the radius $R \forall t \in\left[t_{0}, t_{f}\right]$.

Remark 6.1 By referring to (9) and (12), one can see that the relation between the applied control inputs $v^{i}$ and the nominal one $v^{i *}$ is given by $v^{i}=v^{i *}+$ $\Psi^{i}\left(e^{i}, \dot{e}^{i}\right)$ with $\Psi^{i}\left(e^{i}, \dot{e}^{i}\right)=K_{i 1} \dot{e}^{i}+K_{i 2} e^{i}(i=1,2,3)$. The amplitude of $\Psi^{i}$ depends on model uncertainties and external disturbances. Thus, the nominal control $v^{i *}$ is based on the nominal model (7) whereas the true control input will be based on the real model. To take into consideration model uncertainties, a parameter $\rho$ is introduced in (16) where $0<\rho<1$. This will create a boundary layer to ensure that $v(t)$ inside $\Phi$ regardless model uncertainties:

$$
\left\{\begin{array}{l}
\text { Minimize } t_{f}-t_{0} \\
\text { Subject to } \sum_{i=1}^{3}\left(v^{i *}(t)-C^{i}\right)^{2}<\rho^{2} R^{2} \quad \forall t
\end{array} .\right.
$$


The numerical results shown in the sequel are obtained from the application of the flatness-based trajectory planning and controller developed above to the nonlinear models of the chaser and target in rendezvous mission. Model uncertainties arises then from the mismatch between the Clohessy-Wiltshire model (2) and the nonlinear models of the chaser and target (1). Later on in this work, the domain $\Phi$ is simplified to determine the largest sphere containing the point $V_{s s}$ and included in $\Phi$ which introduces further uncertainties.

The objective of the rendezvous mission is to drive the chaser from an initial position to the position of the target. Thus, the objective is to start with $F^{i}\left(t_{0}\right) \neq 0$ and $\dot{F}^{i}\left(t_{0}\right) \neq 0$ and to end the mission with $F^{i}\left(t_{f}\right)=\dot{F}^{i}\left(t_{f}\right)=0$ $(i=1,2,3)$. The first step in the fault-free trajectory planning consists in determining $C$ and $R$, respectively the centre and the radius of the largest sphere containing $V_{s s}\left(v_{s s}^{1 *}, v_{s s}^{2 *}, v_{s s}^{3 *}\right)$ and included in $\Phi$. To determine this sphere, the analytical approach presented in (Sahu and Lahiri, 2004) is employed. This approach consists in considering each combination of four planes and finding the insphere bounded by the planes. If a polyhedron has $N$ facets, then $C_{4}^{N}$ combinations each containing four planes can be made. As a result, $C_{4}^{N}$ inspheres can be found and the one to consider is the largest one that does not intersect any facet of the polyhedron. Back to the domain $\Phi$, it is a polyhedron of 264 facets; thus resulting in $C_{4}^{264}=197,829,126$ inspheres. Although that the determination of the largest sphere is performed off-line, still the calculation time is huge. To simplify, only the most informative facets are kept by eliminating one of each parallel facets and by eliminating all neighboring facets but one. This results in the simplified domain $\Phi$ given in Figure 1(b). This simplified domain has 28 facets and thus resulting in $C_{4}^{28}=20,475$ inspheres which are easier to calculate. Indeed, the largest sphere obtained with the simplified domain may have intersection with some of the 264 facets. This can be easily taken into consideration by adjusting $\rho$ in (17). The application of the method to the simplified domain $\Phi$ gives the largest insphere illustrated in Figure 1(c). In the second step, problem (17) is solved the obtained solution is $t_{f}=79.82 \mathrm{~s}$. Figure 3(a) shows the relative position between the chaser and the target in the fault-free case. At $t_{0}=0 \mathrm{~s}$, the relative position is different than zero and at $t_{f}=79.82 \mathrm{~s}$, it equals zero. Figure $4(\mathrm{a})$ shows the applied effort $V^{*}(t)$. It can be noted that $V^{*}(t)<R^{2} \forall t \in\left[t_{0}, t_{f}\right]$. Thus $v^{*}(t) \in \Phi \forall t \in\left[t_{0}, t_{f}\right]$ and the desired effort vector can be generated by the actuators without hitting the control bounds $u_{\max }=20 \mathrm{~N}$ as illustrated in Figure 5(a).

\subsubsection{Special case}

The constraint in (17) is a function of time $t$ and solving the trajectory planning problem requires calculus of variations. A more suitable trajectory planning problem for on-board implementation can be obtained when the two following conditions are met:

- The reference trajectory is defined as a Bézier polynomial function of degree three instead of degree five as defined in (8). This results in less smooth control efforts $v^{i}$ for $i=1,2,3$ but facilitates trajectory planning problem.

- The relative distances $F^{i}(i=1,3)$ are small in such a way that the terms 
$3 w^{2} F^{1 *}$ and $w^{2} F^{3 *}$ in (9) can be considered negligible with respect to the other terms.

In this case, define $V_{E x t}^{*}$ as the extrema of $V^{*}(t)=\sum_{i=1}^{3}\left(v^{i *}(t)-C^{i}\right)^{2}$ where the extrema collectively denote the minima and the maxima of $V^{*}(t)$. The extrema can be obtained as follows:

$$
V_{E x t}^{*}=V^{*}\left(t_{E x t}\right)
$$

where $t_{E x t}$ are the critical points, and are the solutions of

$$
\frac{d V^{*}(t)}{d t}=0 .
$$

The three nominal efforts $v^{1 *}, v^{2 *}$ and $v^{3 *}$ can be derived from (7) when replacing the flat output $F^{i}$ by its reference trajectory $F^{i *}$ of degree three. It can be noted that $V^{*}(t)$ is a polynomial of degree six and that $d V^{*}(t) / d t$ is of degree five. Polynomial equations higher than fourth degree are incapable of algebraic solution in terms of a finite number of additions, subtractions, multiplications, divisions, and root extractions. This was shown by Ruffini in 1813 (Wells, 1986). Thus, it is not possible to derive an algebraic solution for $V_{E x t}^{*}$. This problem can be overcome as follows. In (7), the average motion $w$ is generally small (in our application, it equals $8.511 \times 10^{-4}$ ). If the second condition above is met then one can say that $3 w^{2} F^{1 *} \approx 0$ and $w^{2} F^{3 *} \approx 0$. In this case, $V^{*}(t)$ is of degree four and $d V^{*}(t) / d t$ is of degree three. An algebraic solution for $d V^{*}(t) / d t=0$ can then be obtained in function of the initial conditions, the final conditions, the starting time $t_{0}$ and the arriving time $t_{f}$. This algebraic solution is not given here due to its complexity. Finally, the mission time $t_{f}$ that guarantees that $v^{*}(t) \in \Phi \forall t \in\left[t_{0}, t_{f}\right]$ is the solution of the optimization problem

$$
\left\{\begin{array}{l}
\text { Minimize } t_{f}-t_{0} \\
\text { Subject to } V_{E x t}^{*}<\rho^{2} R^{2} ; V^{*}\left(t_{0}\right)<\rho^{2} R^{2} ; V^{*}\left(t_{f}\right)<\rho^{2} R^{2},
\end{array}\right.
$$

where $V^{*}\left(t_{0}\right)$ and $V^{*}\left(t_{f}\right)$ are the expressions of $V^{*}(t)$ at $t_{0}$ and $t_{f}$. The extrema $V_{E x t}^{*}$ are algebraic expressions that are in function of $t_{0}, t_{f}$, the initial and final conditions. Thus, using (20) instead of (17) for trajectory planning may be more adequate for real-time on board implementation: when a planning is required, it is sufficient to plug the parameters ( $t_{0}$, the initial and final conditions) with their numerical values in (17) to get the solution $t_{f}$. Hence, reducing calculation requirements and making the trajectory planner more suitable for on-board implementation.

\subsection{Actuator fault severity}

The chaser's actuators are thrusters that are subject to two major faults: jamming in closed position and jamming in fully open position. When actuator faults occur, the domain $\Phi$ shrinks. This consequently reduces the maneuverability of the system although that it may remain controllable. Each actuator fault will have a different effect according to the actuator location in the system. Thus, the idea is to use the reduction in the domain $\Phi$ to evaluate the severity of faults. The evaluation of faults severity could be crucial in order to determine 
if the desired trajectories should be re-planned or not. When the $i^{\text {th }}$ actuator is faulty, the fault can be accommodated by adding an additional term $-b^{i} \bar{u}^{i}$ to the desired effort vector $v^{*}(t)$. Define $n_{f}$ as the number of occurred faults. It turns out that the total effort to be generated is then:

$$
v_{f}^{*}(t)=\left[\begin{array}{lll}
v_{f}^{1 *} & v_{f}^{2 *} & v_{f}^{3 *}
\end{array}\right]^{T}=v^{*}(t)-\sum_{i=1}^{n_{f}} b^{i} \bar{u}^{i},
$$

where $b^{i}$ is the $i^{\text {th }}$ column of $B$ and $\bar{u}^{i}$ represents the control input of the faulty actuator $\left(\bar{u}^{i}=0\right.$ for an outage fault and $\bar{u}^{i}=u_{\max }$ for a lock in place fault). A negative sign is assigned to $\sum_{i=1}^{n_{f}} b^{i} \bar{u}^{i}$ in (21) to compensate the effect of the fault. In steady state, $v_{s s, f}^{*}=v_{s s}^{*}-\sum_{i=1}^{n_{f}} b^{i} \bar{u}^{i}$.

Two measurements will be employed to evaluate the severity of faults. If $f_{i}$ denotes the fault of the $i^{\text {th }}$ actuator, a first severity measurement $S 1_{f_{i}}$ can then be evaluated as follows:

$$
S 1_{f_{i}}=\frac{V-V_{f_{i}}}{V}
$$

where $V$ is the volume of the nominal domain $\Phi$ and $V_{f_{i}}$ is that of faulty $\Phi_{f}$. This severity measurement evaluates the shrinking of $\Phi$ for the fault $f_{i}$. Thus, $0 \leq S 1_{f_{i}} \leq 1$ and a fault $f_{i}$ is more severe as $S 1_{f_{i}}$ is closer to 1 .

The first severity measurement is not sufficient to evaluate faults severity. This is because $\Phi_{f}$ may still be large but $V_{s s}\left(v_{s s}^{1 *}, v_{s s}^{2 *}, v_{s s}^{3 *}\right)$ is very close to the boundaries. Thus, a second severity measurement $S 2_{f_{i}}$ is defined as follows:

$$
S 2_{f_{i}}=\frac{d-d_{f_{i}}}{d}
$$

where $d$ (respec. $d_{f_{i}}$ ) is the minimal distance from $V_{s s}$ (respec. $V_{s s, f}$ ) to the boundary of $\Phi$ (respec. $\Phi_{f}$ ). This indicator gives information about how close is $V_{s s, f}$ to the boundary of $\Phi_{f}$ : as $V_{s s, f}$ is closer to the boundaries, the chance for $v_{f}^{*}(t)$ to lay outside $\Phi_{f}$ increases.

Remark 6.2 Fault severity is an integrated part of almost all hazard analysis methods used in almost all segments of the industry. The two indicators on fault severity defined above can be evaluated off-line and can serve in many directions. For example, they give an idea on which combinations of actuator faults are the most critical and thus help designers in preventing such combinations. In addition, when working with multi-systems (such as system of subsystems or multi-agents in formation or cooperative control), the fault severity measurements help to better understand how occurred faults reduce the capabilities of the faulty system and consequently affect the global objectives to achieve.

Some actuator fault severity are presented in Table 1. For each fault (jamming in closed and open position), $S 1_{f_{i}}$ and $S 2_{f_{i}}$ are evaluated for the domain $\Phi$. It can be noted that each actuator fault has different severity and that severity increases with multiple faults. Due to the symmetry of $\Phi, S 1_{f_{i}}$ are the same either if the actuator is jammed in closed position or open position. However, $S 2_{f_{i}}$ are greater when actuators are jammed in open position. This is because $V_{s s}\left(v_{s s}^{1 *}, v_{s s}^{2 *}, v_{s s}^{3 *}\right)$ is shifted to $V_{s s, f}\left(v_{s s, f}^{1 *}, v_{s s, f}^{2 *}, v_{s s, f}^{3 *}\right)$ as shown in (21). Figure 2(b) illustrates the domain $\Phi_{f}$ and the largest sphere of centre $V_{s s, f}\left(v_{s s, f}^{1 *}, v_{s s, f}^{2 *}, v_{s s, f}^{3 *}\right)$ with actuators 4,5 and 12 jammed in closed position. 


\begin{tabular}{lcccc} 
& \multicolumn{2}{c}{ Closed position } & \multicolumn{2}{c}{ Open position } \\
\cline { 2 - 5 } Actuator faults & $S 1_{f_{i}}$ & $S 2_{f_{i}}$ & $S 1_{f_{i}}$ & $S 2_{f_{i}}$ \\
No fault & 0 & 0 & 0 & 0 \\
Actuator 1 & 0.238 & 0.345 & 0.238 & 0.698 \\
Actuator 2 & 0.224 & 0.364 & 0.224 & 0.734 \\
Actuator 3 & 0.288 & 0.343 & 0.288 & 0.757 \\
Actuators 1 \& 11 & 0.446 & 0.716 & 0.446 & 1 \\
Actuators 4, 5 \& 12 & 0.616 & 0.731 & 0.616 & 1 \\
Actuators 1, 9 \& 11 & 0.664 & 1 & 0.664 & 1
\end{tabular}

Table 1: Severity of some actuator faults

Compared to Figure 2(a), fault occurrence reduces in huge amount the domain of attainable efforts. It is also important to note how the domain boundaries approach the point $V_{s s, f}$ which increases the chance of the desired efforts $v_{f}^{*}$ to lay outside the attainable domain.

For the fault scenario, the $3^{\text {rd }}$ actuator is assumed to jam in open position. The fault occurs at time instant $30 \mathrm{~s}$. Figure 3(b) illustrates the fault effect on the system's behavior. It is clear that the chaser is not able anymore to reach the target. The controller tries to reach the trajectory but generates a desired effort that lays outside the attainable effort domain (Figure 4(b)) and thus it cannot be generated by the actuators that hit their control bounds (Figure $5(\mathrm{~b})$ ). Figure 5(b) shows the control input of the $3^{r d}$ actuator jammed in open position at $t=30 \mathrm{~s}$.

\subsection{Control re-allocation against control re-allocation with trajectory re-planning}

In a first step, control re-allocation is applied at $t_{\text {accom }}=35 \mathrm{~s}\left(t_{\text {accom }}\right.$ is the time of fault accommodation) to accommodate the actuator fault where 5 seconds are assumed to be taken by the fault detection and diagnosis module. This strategy is able to improve the system's performance (Figure 3(c)) compared to that without accommodation. However, the chaser is still not able to reach the target and some control inputs are saturated (Figures 4(c) and 5(c)).

In a second step, the trajectory re-planning is combined with control reallocation. Similarly to control allocation, the control re-allocation is achieved by using direct allocation formulated as linear programming problem (Bodson, 2002). In this case, the $3^{r d}$ column of matrix $B$ is removed and the objective is to

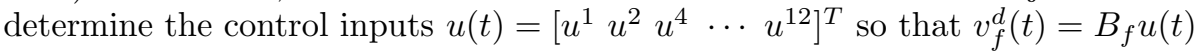
where $B_{f}$ is matrix $B$ without the $3^{r d}$ column $b^{3}$ and $v_{f}^{d}(t)$ is the vector of desired control efforts in the fault case and it is given as $v_{f}^{d}(t)=\left[v_{f}^{1 d} v_{f}^{2 d} v_{f}^{3 d}\right]^{T}=v^{d}(t)-$ $b^{3} \bar{u}^{3}$ as defined in (21). In this case, an actuator fault can be accommodated if it is always possible to drive the system to the final state $F_{f}$. This is possible if $v_{f}^{*}(t) \in \Phi_{f} \forall t \geq t_{\text {accom }}$. This is simply another way to say that a fault can be accommodated if there are sufficient remaining resources. Many of the existing FTC methods do not guarantee that the desired efforts $v_{f}^{*}(t)$ will remain inside $\Phi_{f} \forall t \geq t_{\text {accom }}$ especially when $\Phi$ shrinks and $V_{s s, f}$ is close to the boundaries of $\Phi_{f}$. If the desired effort $v_{f}^{*}(t) \notin \Phi_{f}$, the control inputs $u$ that verify the relation $B u(t)=v_{f}^{*}(t)$ do not lie in $\Omega_{f}$ (for the faulty case) and thus cannot 
be generated by the actuators. This results in degraded performance and may lead in some cases to instability when actuators hit their limits. It is obvious that a better FTC can be achieved with a trajectory re-planning taking into consideration the remaining resources. For this purpose, problem (17) can be employed by considering the new system post-fault conditions: the two step trajectory planning is repeated but this time with application to $\Phi_{f}$. The faulttolerance control strategy combining control re-allocation and trajectory replanning is applied at $t_{\text {accom }}=35 \mathrm{~s}$. The largest sphere included in $\Phi_{f}$ of centre $C_{f}$ and of radius $R_{f}$ is determined. Solving the optimization problem with the new initial conditions of the system (at $t_{a c c o m}=35 \mathrm{~s}$ ) gives $t_{f}=317.6 \mathrm{~s}$. The simulation shows that re-planning system trajectory combined with control reallocation allows the system to reach the target (Figure 3(d)) without hitting the control bounds (Figures $4(\mathrm{~d})$ and $5(\mathrm{~d})$ ). Because of the severity of the fault, $t_{f}=317.6 \mathrm{~s}$ is obtained at the re-planning.

\section{Conclusion}

This paper proposes a trajectory planning/re-planning approach for over-actuated systems by using flatness. For illustration, the approach is applied to satellite systems in rendezvous mission but can be applied to other flat systems. The ideas of this work can be summarized as follows:

1. Propose a method to evaluate the severity of faults based on the shrinking of the domain of attainable efforts.

2. Formulate the trajectory planning/re-planning approach as an optimization problem where the objective is to tune the total mission time $t_{f}$ so as to reach the desired objectives as fast as possible without hitting actuator constraints.

3. In some conditions, it is possible to replace the constraints of the optimization problem (which are explicitly functions of time $t$ ) with their extrema (which are independent of $t$ ). This reduces calculation requirements and makes the trajectory planning problem more suitable for real-time onboard implementation.

4. Replace the domain of attainable efforts with simple geometric shapes to facilitate solving the optimization problem.

As pointed out before, the obtained trajectories are suboptimal for two reasons. First, the reference trajectories are restricted to polynomial functions of time $t$ and second, the domain $\Phi$ of attainable control effort $v$ is replaced by a spherical subset. Several issues should be investigated in future works:

1. This paper considers the position dynamics of two satellite systems in rendezvous mission. In reality, the rendezvous mission consists also of the attitude (rotation) dynamics. Those dynamics are not considered in this paper for two reasons. First, the attitude dynamics are nonlinear and thus it will be more difficult to apply the proposed approach to them. Second, considering both position and attitude dynamics means that vector $v \in \Re^{6}$ which is impossible to represent geometrically. One possible solution is to linearize (if possible) the attitude dynamics and to break down $v$ into two vectors $v_{1} \in \Re^{3}$ and $v_{2} \in \Re^{3}$. 
2. The FTC system requires information about the location of the faulty actuator, the amplitude and the type of the fault. It is assumed here that an FDI module is present to detect and isolate faults.

3. This paper formulates the trajectory planning as an optimization problem with one decision variable: the final time of the mission $t_{f}$. In some cases, only changing $t_{f}$ may not sufficient and thus changing the final state $F_{f}$ may also be necessary.

\section{References}

Bemporad, A., Casavola, A., and Mosca, E. (1997), "Nonlinear control of constrained linear systems via predictive reference management," IEEE Transactions on Automatic Control, 42(3), 340-349.

Blanke, M., Kinnaert, M., Lunze, J., and Staroswiecki, M., Diagnosis and faulttolerant control, Springer (2006).

Bodson, M. (2002), "Evaluation of optimization methods for control allocation," Journal of Guidance, Control, and Dynamics, 25(4), 703-711.

Bordignon, K.A. (1996), "Constrained control allocation for systems with redundant control effectors," Ph.D. dissertation, Virginia Polytechnic Institute and State University.

Bouktir, Y., Haddad, M., and Chettibi, T. (2008), "Trajectory planning for a quadrotor helicopter," in 16th Mediterranean Conference on Control and Automation, 25-27 June, Ajaccio, Corsica, France, pp. 1258-1263.

Castelan, E., da Silva Jr., J.G., and Cury, J. (1996), "A reduced-order framework applied to linear systems with constrained controls," IEEE Transactions on Automatic Control, 41(2), 249-255.

Cowling, I., Whidborne, J., and Cooke, A. (2006), "Optimal trajectory planning and LQR control for a quadrotor UAV," in Proceedings of the UKACC International Conference on Control, Glasgow, United Kingdom.

Dardinier-Maron, V., Hamelin, F., and Noura, H. (1999), "A fault-tolerant control design against major actuators failures: application to a three-tank system," in Conference on Decision and Control, Phoenix, AZ , USA, 7-10 December, pp. 3569-3574.

Dong, Q., Zhong, M., and Ding, S.X. (2010), "Active fault tolerant control for a class of linear time-delay systems in finite frequency domain," International Journal of Systems Science, 43(3), 543-551.

Fliess, M., Lévine, J., Martin, P., and Rouchon, P. (1995), "Flatness and defect of nonlinear systems: introductory theory and example," International Journal of Control, 61(6), 1327-1361.

Gensior, A., Woywode, O., Rudolph, J., and Güldner, H. (2006), "On differential flatness, trajectory planning, observers, and stabilization for DCDC converters," IEEE Transactions on Circuits and Systems-I: Regular Papers, 53(9), 2000-2010. 
Gilbert, E., and Kolmanovsky, I. (2002), "Nonlinear tracking control in the presence of state and control constraints: a generalized reference governor," Automatica, 38(12), 2063-2073.

Henrion, D., Tarbouriech, S., and Kučera, V. (2001), "Control of linear systems subject to input constraints: a polynomial approach," Automatica, 37, 597604.

Hsieh, C.S. (2002), "Performance gain margins of the two-stage LQ reliable control," Automatica, 38(11), 1985-1990.

Mai, P., Join, C., and Reger, J. (2007), "Flatness-based fault-tolerant control of a nonlinear MIMO system using algebraic derivative estimation," in Preprints of the 3rd IFAC Symposium on System, Structure and Control, Foz do Iguassu, Brazil, 17-19 October.

Noura, H., Theilliol, D., Ponsart, J.C., and Chamseddine, A., Fault-tolerant control systems: Design and practical applications, Springer London (2009).

Petersen, J., and Bodson, M. (2006), "Constrained quadratic programming techniques for control allocation," IEEE Transactions on Control Systems Technology, 14(1), 91-98.

Ross, I., and Fahroo, F. (2006), "Issues in the real-time computation of optimal control," Mathematical and Computer Modelling, 43(9-10), 1172-1188.

Rudolph, J., and Winkler, J. (2003), "A Generalized flatness concept for nonlinear delay systems: motivation by chemical reactor models with constant or input dependent delays," International Journal of Systems Science, 34(8-9), 529-541.

Sahu, K., and Lahiri, A. (2004), "Finding the insphere of a convex polyhedron: an analytical approach," Philosophical Magazine, 84(12), 1185-1196.

SIRASAS,, "https://extranet.ims-bordeaux.fr/External/SIRASAS/", [cited 24 November 2011] (2011).

Suryawan, F., Doná, J.D., and Seron, M. (2012), "Splines and polynomial tools for flatness-based constrained motion planning," International Journal of Systems Science, 43(8), 1396-1411.

Tarbouriech, S., Garcia, G., and da Silva Jr., J.G. (2002), "Robust stability of uncertain polytopic linear time-delay systems with saturating inputs: an LMI approach," Computers and Electrical Engineering, 28(3), 157-169.

Theilliol, D., Zhang, Y., and Ponsart, J.C. (2009), "Fault tolerant control system against actuator failures based on re-configuring reference input," in $A d$ vances in Computational Tools for Engineering Applications, Zouk Mosbeh, Lebanon, 15-17 July, pp. 480-485.

Wells, D., The penguin dictionary of curious and interesting numbers, Middlesex, England: Penguin Books (1986). 
Zhang, Y., and Jiang, J. (2003), "Fault tolerant control system design with explicit consideration of performance degradation," IEEE Transactions on Aerospace and Electronic Systems, 39(3), 838-848.

Zhang, Y., and Jiang, J. (2008), "Bibliographical review on reconfigurable faulttolerant control systems," Annual Reviews in Control, 32(2), 229-252. 


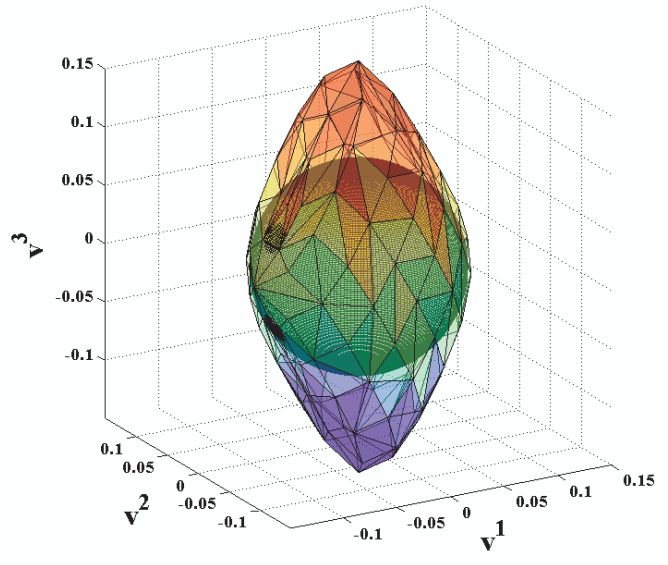

(a) $\Phi$ and largest insphere

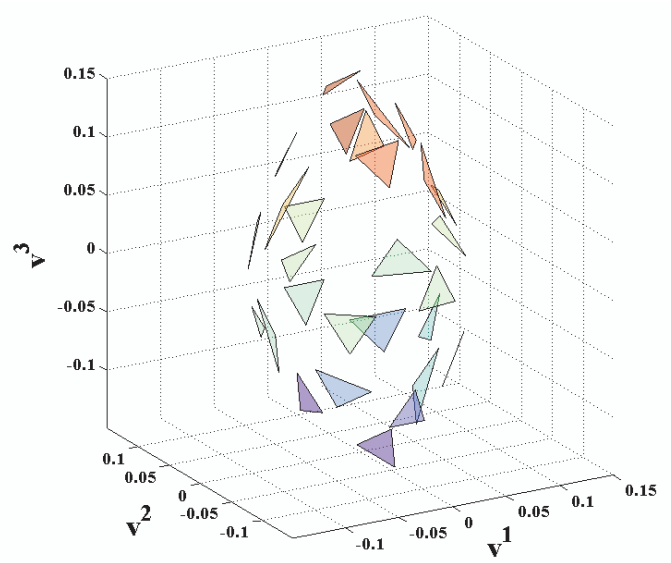

(b) Simplified domain $\Phi$

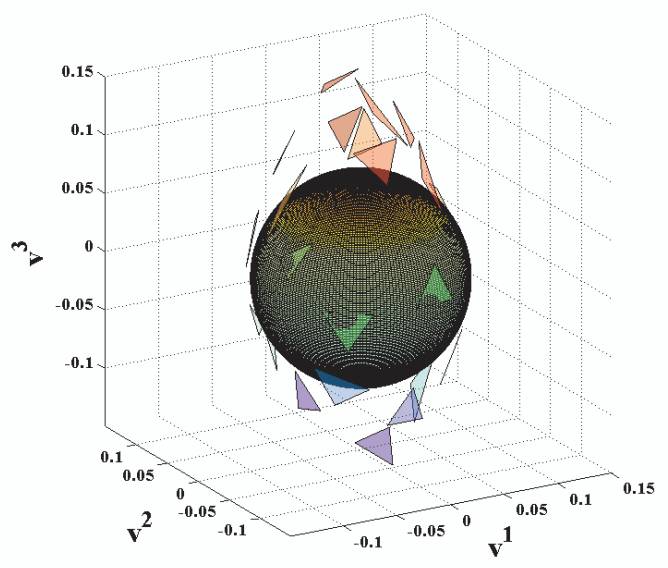

(c) Simplified $\Phi$ and largest insphere

Figure 1: Domain $\Phi$ and largest inspheres in the fault-free case 


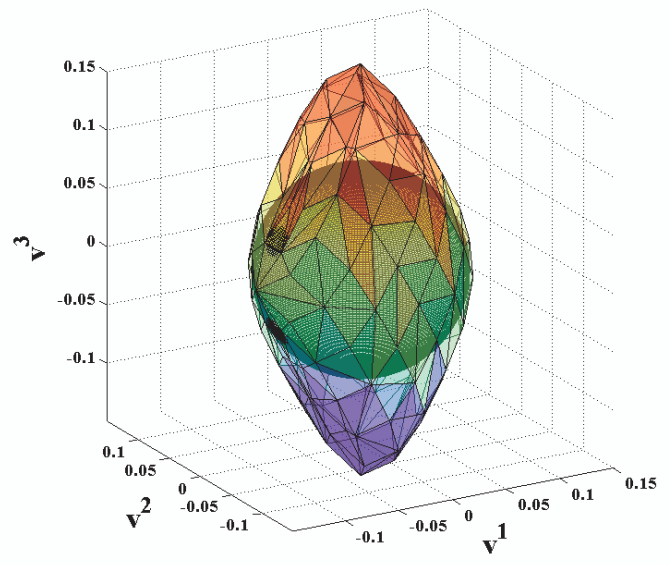

(a) Simplified $\Phi$ and largest insphere

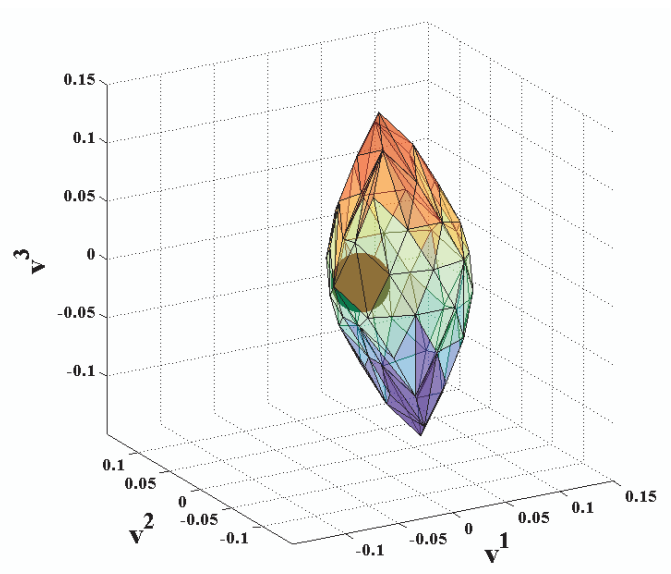

(b) $\Phi_{f}$ in the fault case

Figure 2: Domain $\Phi$ and largest inspheres in the fault case 


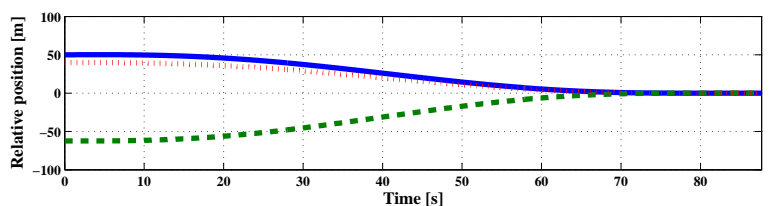

(a) Fault-free case

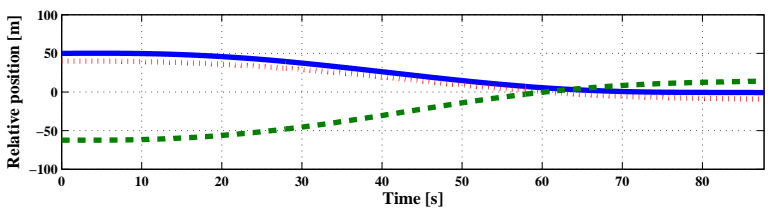

(c) Fault case with control re-allocation

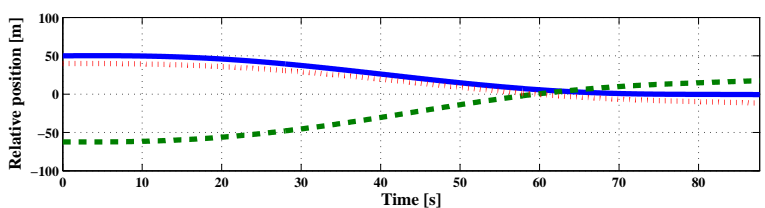

(b) Fault case

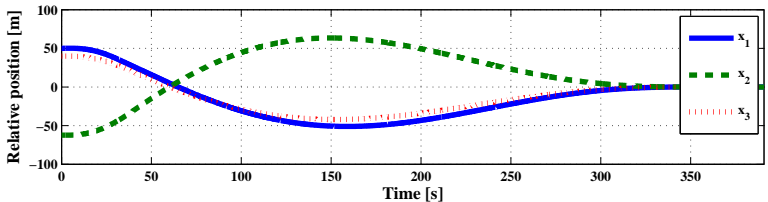

(d) Fault case with control re-allocation and replanning

Figure 3: The relative position of the chaser with respect to the target

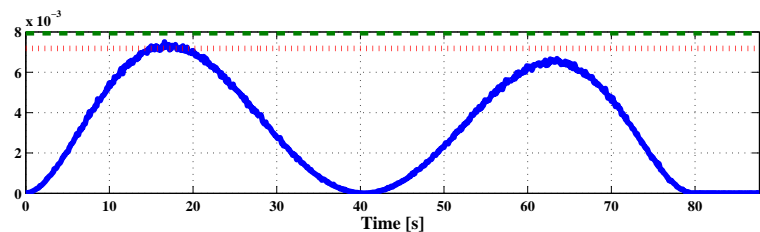

(a) Fault-free case

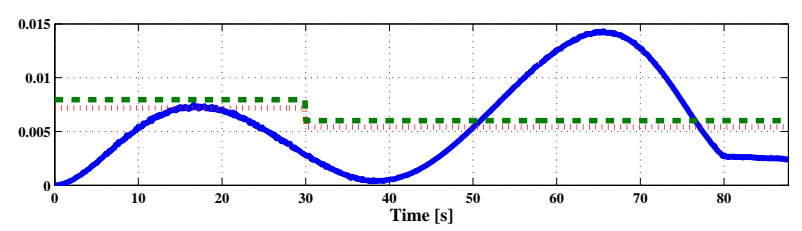

(c) Fault case with control re-allocation

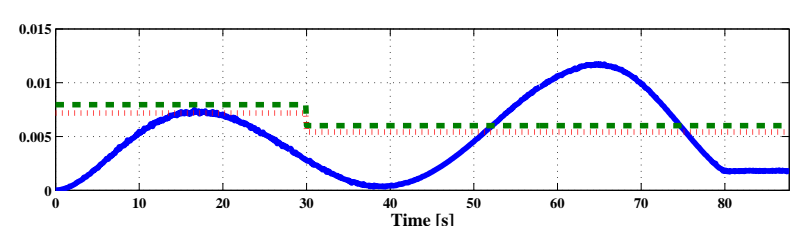

(b) Fault case

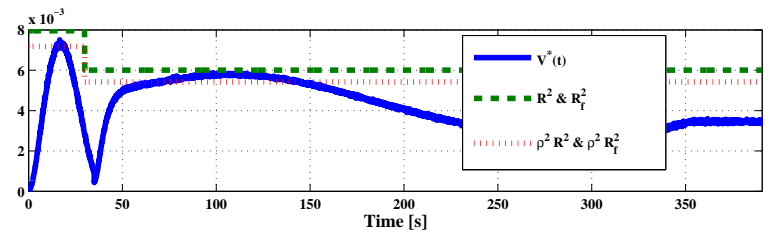

(d) Fault case with control re-allocation and replanning

Figure 4: The distance between $v^{*}(t)$ and $C: V^{*}(t)=\sum_{i=1}^{3}\left(v^{i *}(t)-C^{i}\right)^{2}$ 


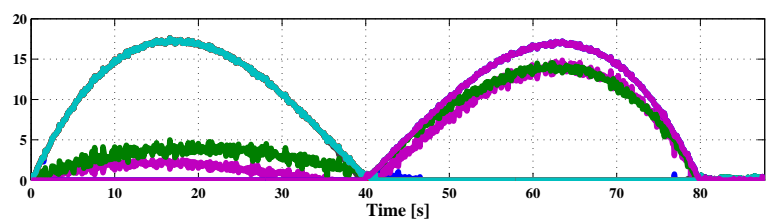

(a) Fault-free case

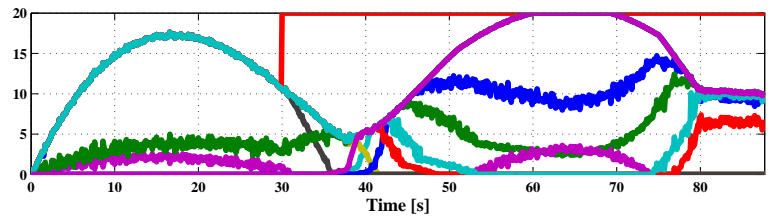

(c) Fault case with control re-allocation

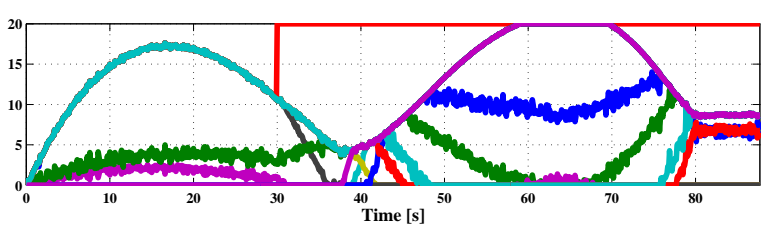

(b) Fault case

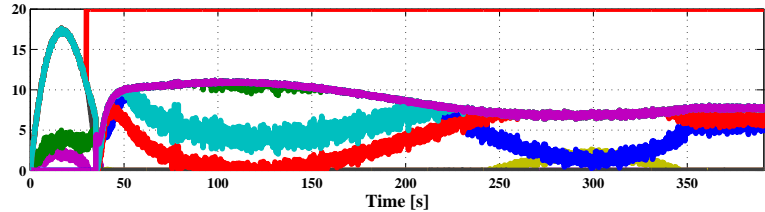

(d) Fault case with control re-allocation and replanning

Figure 5: The control inputs $u^{i}(i=1, \cdots, 12)$ 